\title{
Modelos Cosmológicos e a Aceleração do Universo
}

(Cosmological Models and the Acceleration of the Universe)

\author{
Helio V. Fagundes \\ Instituto de Física Teórica, Universidade Estadual Paulista, \\ São Paulo - SP - CEP 01405-900 \\ E-mail: helio@ift.unesp.br
}

Recebido em 2 dezembro, 2001. Aceito em 8 de maio, 2002.

\begin{abstract}
Alguns modelos cosmológicos são apresentados informalmente, especialmente os de Friedmann e Einstein-de Sitter, utilizados no contexto do chamado 'Big Bang', e os de Friedmann-Lemaitre que completam os primeiros por incluir a aceleração da expansão do universo, descoberta alguns anos atrás.
\end{abstract}

\section{O universo observável}

A Fig. 1 é um esquema da parte do universo que podemos observar da Terra. Os círculos representam camadas esféricas, com um número indicando sua distância em anos-luz. Ela já pressupõe interpretações teóricas, ou ao menos algum processamento mental, pois nossos sentidos não percebem diretamente distâncias astronômicas. Nossa visão direta do céu não indica profundidade na direção da observação: o Sol, a Lua, as estrelas $\alpha$ e $\beta$ do Cruzeiro do Sul (distantes respectivamente 260 e 490 anos-luz) aparentam estar todos à mesma distância de nós.

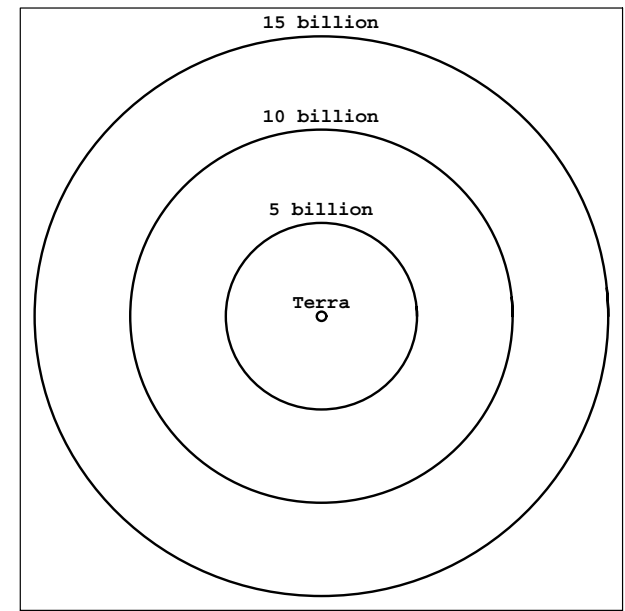

Figura 1. Esquema da parte do universo que podemos observar. Cada círculo representa uma camada esférica, com a distância indicada em anos-luz.

Consideremos o círculo marcado 5 bilhões ( 1 bilhão $=1000$ milhões) na figura. Os objetos, como galáxias e quasares, situados nessa camada, são observados por nós na forma que tinham 5 bilhões de anos atrás. Ou seja, cada círculo representa uma região do cosmo em uma certa época. (Mas na escala de hoje, que leva em conta a expansão do espaço.) Não podemos ver como era em qualquer época uma dada região. O círculo externo é a parte mais distante, a cerca de 15 bilhões de anos-luz, e portanto mais antiga, que pode ser observada através de radiação eletromagnética.

Os números da Fig. 1 são aproximados, mas todos se baseiam na teoria da expansão do universo, conhecida como 'Big Bang' (BB), ou, tecnicamente, como teoria padrão do universo. Outras observações que são explicadas pela teoria do BB são as densidades relativas dos elementos leves, hidrogênio, hélio e lítio, no universo, e a chamada radiação cósmica de fundo, que se origina na esfera máxima da Fig. 1.

Acontece que o $\mathrm{BB}$ tem muitas variantes. A idéia básica do cosmo como algo semelhante ao resultado de uma grande explosão continua sendo a mais aceita pelos especialistas, mas quanto aos detalhes há muitas possibilidades e incertezas.

Antes de abordar esses modelos, uma observação: não confundir a cosmologia relativística com a teoria da relatividade geral criada por A. Einstein em 1915. Esta última é uma teoria da força gravitacional, enquanto a cosmologia einsteiniana baseia-se na relatividade geral combinada com outras hipóteses, como a homogeneidade e a isotropia da distribuição de matéria em grandes escalas e a equação de estado dessa matéria. Estas hipóteses adicionais podem ser mais ou menos verdadeiras, daí a variedade de modelos todos baseados na relatividade geral. 


\section{Os modelos de Friedmann e de Einstein-de Sitter}

A. Friedmann, usando as hipóteses de homogeneidade e isotropia da matéria, construiu dois modelos em que a geometria do espaço cósmico (tecnicamente, a geometria das seçôes espaciais 3-dimensionais do espaçotempo 4-dimensional) tem curvatura positiva (fechado, 1922) ou negativa (aberto, 1924). A curvatura é uma quantidade geométrica que vem da teoria das superfícies curvas. Destas, a mais simples é a esfera, que tem curvatura igual ao inverso do raio ao quadrado. Veremos, a seguir, uma caracterização dessas geometrias em termos de triângulos retilíneos.

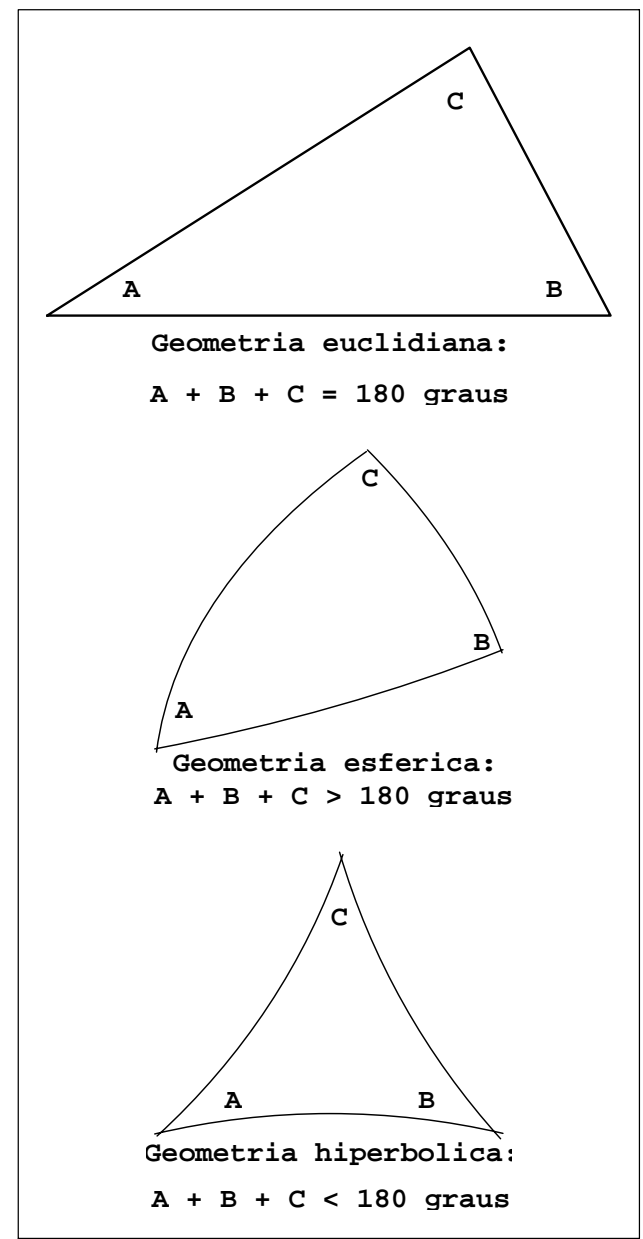

Figura 2. Triângulo "retilíneo" nas três geometrias de curvatura constante.

No século XIX haviam sido descobertas as chamadas geometria hiperbólica e geometria esférica, diferentes da geometria euclidiana comum. Um teorema bem conhecido da geometria euclidiana, que tem curvatura nula, diz que a soma dos ângulos internos de um triângulo retilíneo é igual a 180 graus. Na geometria esférica essa soma é maior que 180 graus, e na geometria hiperbólica ela é menor que 180 graus. A Fig. 2 representa o triângulo euclidiano corretamente, dentro das limitações do desenho. Para os triângulos esférico e hiperbólico as ilustrações são distorcidas, por causa da curvatura: os lados representados por curvas são geodésicas - que são as "retas" nessas geometrias, por exemplo o equador e os meridianos num globo terrestre.

\section{II.1. Modelo de Friedmann esférico ou fechado}

Nesta versão a densidade média de matéria é maior que uma certa densidade crítica da teoria, que corresponde a cerca de cinco vezes a massa de um átomo de hidrogênio por metro cúbico. Usa-se a letra grega $\Omega$ (ômega) para a densidade de matéria em unidades da densidade crítica. Portanto, $\Omega$ é um número maior do que um no modelo esférico. A seção espacial ou 3espaço é representado por uma hiperesfera: assim como a esfera é uma superfície finita, de duas dimensões (latitude e longitude), imersa no espaço euclidiano comum, a hiperesfera é uma hipersuperfície finita, de três dimensões (distância, latitude e longitude), imersa em um espaço euclidiano abstrato de quatro dimensões, que não é intuitivo. Vamos nos limitar a considerar a esfera comum, que seria um "plano" na hiperesfera.

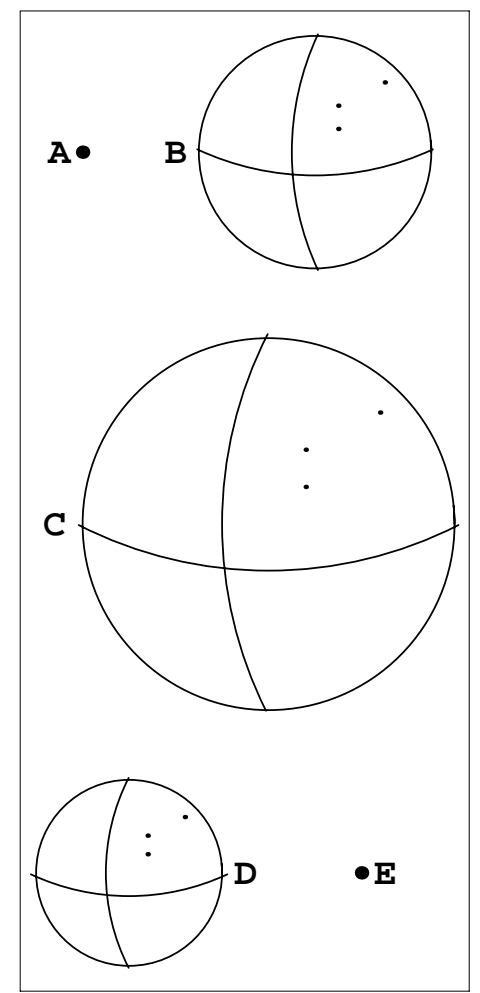

Figura 3. Expansão e contração no modelo de Friedmann esférico: $\mathrm{A} \rightarrow \mathrm{B} \rightarrow \mathrm{C} \rightarrow \mathrm{D} \rightarrow \mathrm{E}$. Em $\mathrm{B}, \mathrm{C}, \mathrm{D}$ os pequenos pontos representam galáxias. 
A Fig. 3 representa grosseiramente a evolução deste modelo, por meio de um balão esférico cujo raio inicialmente (A) tem um diâmetro mínimo ("quase nulo"), expande-se até $\mathrm{B}$ e chega a um valor máximo em $\mathrm{C}$, para depois se contrair até $\mathrm{D}$, e se extinguir em E, novamente com raio mínimo. Em $\mathrm{B}, \mathrm{C}, \mathrm{D}$ as manchas sobre o balão representam estruturas como galáxias ou seus aglomerados. As distâncias entre esses objetos é proporcional ao raio do balão, isto é, primeiro aumentam e depois se contraem. Na época atual, estaríamos no estágio B, portanto em estado de expansão.

\section{II.2. Modelo de Friedmann hiperbólico ou aberto}

Neste caso, densidade média é menor que a densidade crítica, portanto $\Omega$ é uma fração da unidade. $O$ análogo da Fig. 3 é a Fig. 4. A expansão continua sempre, embora com velocidade cada vez menor, ou seja, a expansão é desacelerada ou freada. A seção espacial é o espaço hiperbólico infinito. Aqui no IFT temos trabalhado com uma variante deste modelo, onde o espaço é também hiperbólico, porém finito.

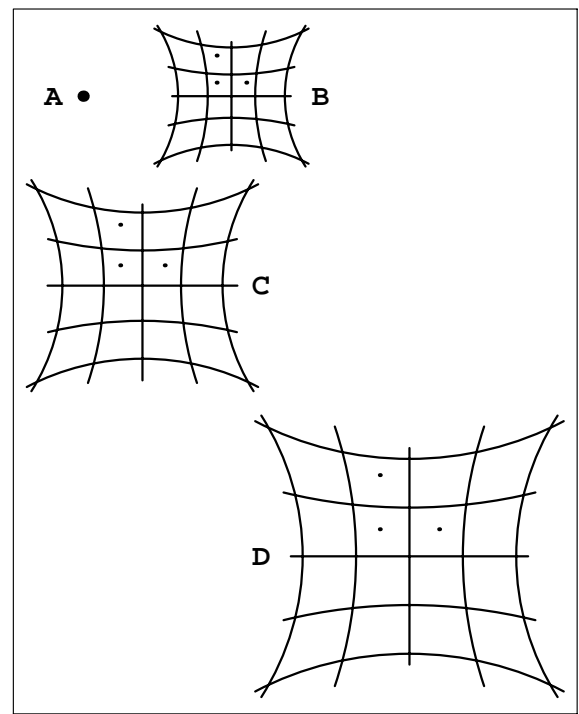

Figura 4. Expansão no modelo de Friedmann hiperbólico: $A \rightarrow B \rightarrow C \rightarrow D \rightarrow \ldots$

\section{II.3. Modelo de Einstein-de Sitter}

Este modelo (1932) supõe que a densidade de matéria é exatamente a densidade crítica, portanto que $\Omega=1$. Sua evolução é representada na Fig. 5. O espaço 3-dimensional (seção espacial) é agora o espaço euclidiano infinito.

Com relação às Figs. 3 a 5 , costuma-se dizer que as galáxias permanecem fixas no espaço, enquanto este se dilata ou contrai; por isso as primeiras afastam-se ou aproximam-se umas das outras.

O modelo de Einstein-de Sitter é, às vezes, chamado modelo de Friedmann de curvatura nula. Ou seja, a expressão modelos de Friedmann pode designar as três teorias que acabamos de descrever. É um mistério para mim por que o próprio Friedmann não tratou do caso euclidiano, que é o mais simples dos três.

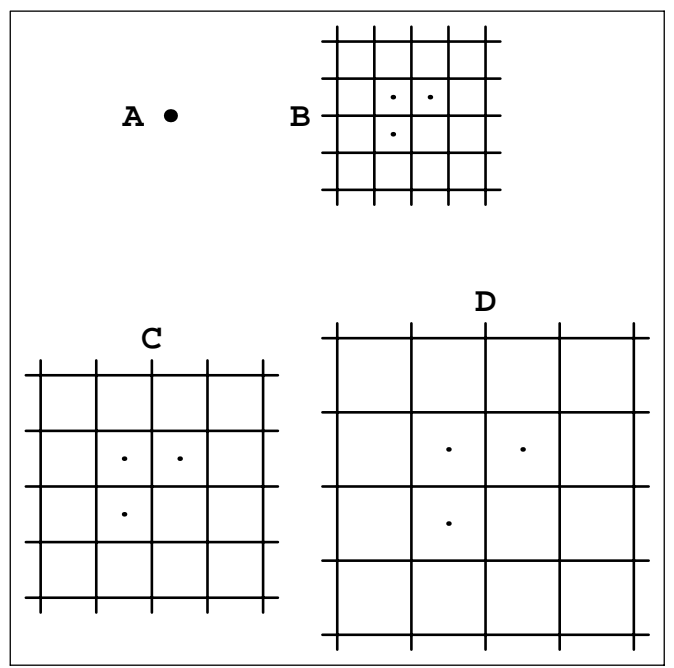

Figura 5. Expansão no modelo de Einstein-de Sitter: $A \rightarrow$ $B \rightarrow C \rightarrow D \rightarrow \ldots$

Na Fig. 6, vemos um gráfico da evolução do fator de escala ou de expansão nos três modelos de Friedmann. Esse fator mede o grau de expansão ou contração. Por exemplo, se em certa época o fator é o duplo de seu valor em outra época, então a distância entre duas galáxias na primeira é o duplo da distância entre elas na segunda. Notem que as três linhas se curvam para baixo, o que matematicamente significa desaceleração. Esta é produzida pela atração gravitacional das partes do universo entre si.

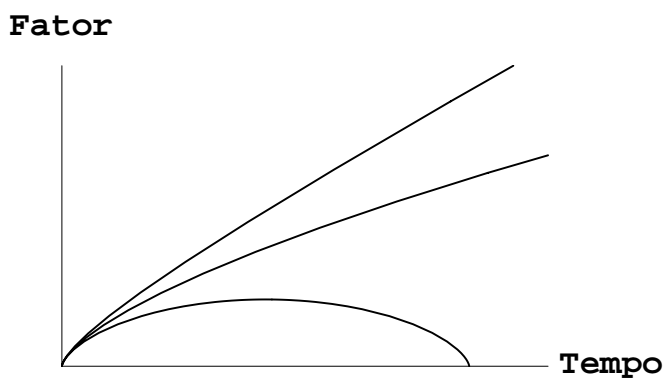

Figura 6. Fator de escala nos modelos de Einstein-de Sitter (a curva do meio), de Friedmann esférico (em baixo) e Friedmann hiperbólico (no topo).

\section{II.4. A lei de Hubble}

O afastamento das galáxias previsto por Friedmann foi confirmado pelas observações, realizadas por $\mathrm{V}$. Slipher, do deslocamento para o vermelho (ou redshift) da luz proveniente das galáxias, e pelas distâncias de galáxias medidas por E. Hubble, ambos resultados publicados na década de 1920. Em 1928, H. Robertson formulou a lei que ficou conhecida como lei de Hubble: a 
velocidade de afastamento das galáxias é proporcional à sua distância. Essa lei exprime a chamada expansão do universo, que os três modelos acima prevêem, mas com diferenças nos valores numéricos. Notar nas Figs. de 3 a 5 que esse afastamento das galáxias não é só com relação a nós na Terra, mas de todas as galáxias entre si.

Em 1948, foi proposta por H. Bondi e T. Gold uma teoria estacionária do cosmo, que também previa a expansão mas não o BB. Ela foi praticamente abandonada com a comprovação, em 1965, da previsão da radiação de fundo, que é explicada de modo natural nos modelos BB. Também contribuiu para isso a confirmação da previsão destes quanto à abundância do elemento químico hélio no universo.

Os três modelos acima, de Friedmann e Einsteinde Sitter, todos prevêm uma expansão do universo que está desacelerando. Ou seja, a velocidade da expansão está sempre diminuindo. Isso se deve a que a única força atuante é a atração gravitacional, que freia a grande grande velocidade inicial. Até há poucos anos, os teóricos achavam que esta previsão estava correta, e apenas se dividiam em duas preferências principais: uma corrente, ligada aos astrofísicos, acreditava numa baixa densidade de matéria, algo como $30 \%$ da densidade crítica, e que portanto o modelo apropriado era o de Friedmann hiperbólico; outra, principalmente dos físicos trabalhando na teoria dos campos, preferia a de Einstein-de Sitter.

Então, três anos atrás, apareceu um novo resultado, espetacular já que ninguém o esperava. Da observação de estrelas supernovas distantes, dois grupos independentes, liderados por A. G. Riess e S. Perlmutter, chegaram à conclusão de que a expansão do universo não está freando, ela está acelerando! (Na seção 4, adiante, teremos uma noção do princípio desta descoberta.) Iniciou-se, então, a atual fase de frenética pesquisa para adaptar o BB a esse novo fato.

Por que tanto alvoroço diante deste novo fato? Para o cientista não se trata de uma observação isolada, é, antes, mais uma peça na investigação das leis naturais. E para todos nós, habitantes do Universo, é importante conhecer os detalhes desta nossa casa!

\section{A constante cosmológica e os modelos de Lemaitre e de Sitter}

Em 1917, quando ainda não se conhecia a expansão do universo, Einstein construiu um modelo cosmológico estático. Para isso ele usou sua própria teoria da gravitação, "com uma pequena modificação". Esta modificação foi acrescentar, na equação do campo gravitacional, a chamada constante cosmológica, o que equivale a acrescentar à densidade de matéria uma outra densidade de energia invisível, que pode ser chamada energia escura, ou energia do vácuo. Seu valor foi ajustado de modo a funcionar como uma força repulsiva e assim contrabalançar a atração gravitacional da matéria comum, permitindo modelar um cosmo sem expansão nem contração. Essa constante, designada pela letra grega $\Lambda$ (lambda), ficou famosa pela frase de Einstein "a maior burrada de minha vida": sem a modificação ele poderia ter previsto a expansão cósmica, detectada onze anos depois. Por outro lado $\Lambda$ tinha fundamento matemático sólido; tanto assim que foi usada em dois modelos, descritos abaixo, que por muito tempo só tiveram interesse teórico formal, mas que atualmente fazem parte da pesquisa de fronteira.

O modelo de W. de Sitter (1917) não contém matéria, mas apenas uma constante cosmológica. Seu fator de expansão cresce exponencialmente (isto é, muito rapidamente), e o espaço é euclidiano. Na década de 1980, apareceu o chamado cenário inflacionário do universo primordial, que supõe uma época muito curta, logo após o nascimento do universo, em que este se expandiu de forma muitíssimo rápida, segundo o modelo de Sitter, antes de começar a fase de expansão 'normal', que é descrita pelos modelos de Friedmann e seus sucessores. Na Fig. 7 vemos a evolução do modelo de de Sitter, com expansão sempre acelerada: no gráfico, curvatura para cima.

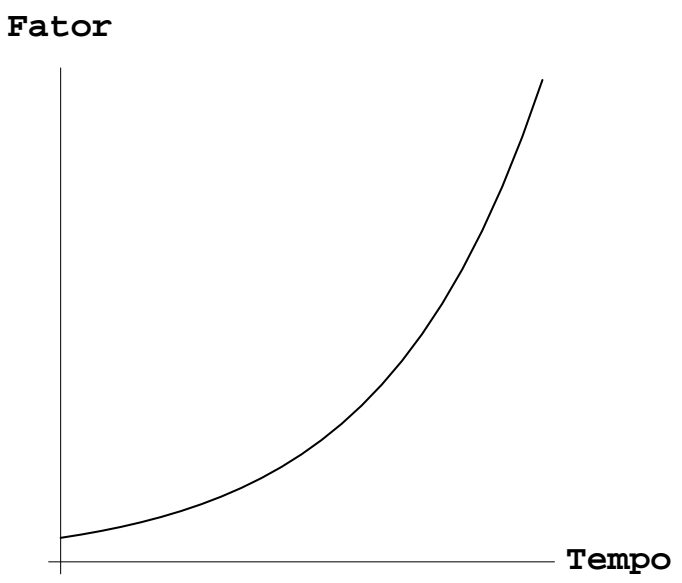

Figura 7. Expansão exponencial no modelo de de Sitter.

No modelo original de G. Lemaitre (1927), a constante cosmológica tem um valor maior que no de Einstein. Ele tem expansão, primeiramente desacelerada, depois acelerada, com uma época de "hesitação" no meio, como diz Harrison em seu excelente livro semipopular. Generaliza-se o modelo original para quaisquer valores de $\Lambda$, e resultam as mesmas variantes geométricas dos modelos de Friedmann, que podem ser chamadas modelos de Friedmann-Lemaitre (FL). Na Fig. 8, temos um gráfico do fator de evolução de algumas das variantes de Friedmann-Lemaitre, todas com $\Lambda$ positivo e, no caso esférico, $\Lambda$ maior que o valor usado 
por Einstein; o que significa uma força repulsiva suficiente para vencer a atração gravitacional, daí a aceleração final da expansão. Para $\Lambda$ menor que o valor de Einstein no caso esférico, e $\Lambda$ negativo em qualquer caso - atração extra! - temos apenas desaceleração e o colapso final, como no Friedmann esférico.

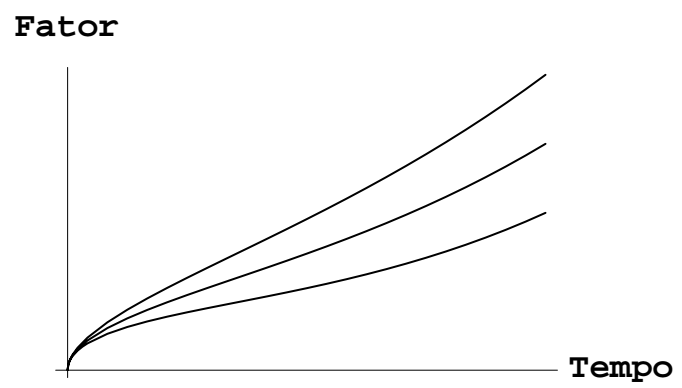

Figura 8. Expansão em modelos de Friedmann-Lemaitre com constante cosmológica positiva, e, no caso esférico, maior que no modelo estático de Einstein. De baixo para cima, espaços esférico, euclidiano e hiperbólico.

Com a descoberta da aceleração da expansão - que ainda não foi suficientemente confirmada - a constante $\Lambda$ voltou à pesquisa de fronteira, como um dos possíveis recursos para encaixar essa aceleração no esquema do $\mathrm{BB}$.

\section{Modelos incluindo a acelera- ção}

Nos três modelos de Friedmann, a expansão é sempre freada, isto é, não prevêem aceleração. No de de Sitter ela é acelerada demais, só serve para descrever a chamada fase inflacionária do universo recém-nascido.

Por outro lado, o esquema inflacionário mais popular é aquele em que a inflação elimina qualquer curvatura que o universo pudesse ter originalmente, de modo que a época pós-inflacionária tem curvatura nula.

O modelo mais simples para acomodar tanto o BB com curvatura nula como a aceleração é o de Friedmann-Lemaitre com espaço euclidiano. À medida que o universo se expande, a densidade de matéria diminui, pois a quantidade de matéria em uma certa região se conserva enquanto o volume da região aumenta. Temos, então, um gráfico da evolução das densidades, em unidades da densidade crítica atual, de matéria e de energia escura como na Fig. 9. Nesta figura notamos que no início predominava a matéria, e assim o universo se parece com o de Einstein-de Sitter (EdS). Eventualmente a energia do vácuo vai predominar, e o modelo se comportará como o de de Sitter $(\mathrm{dS})$.

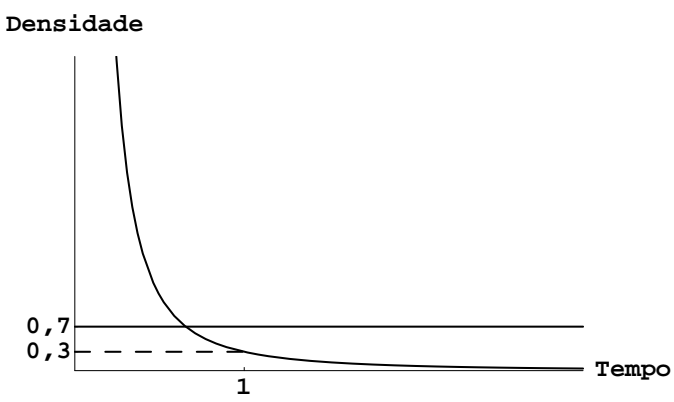

Figura 9. Densidades de matéria (curva) e energia escura (reta), em unidades da densidade crítica atual. O número 1 no eixo do tempo indica a época atual.

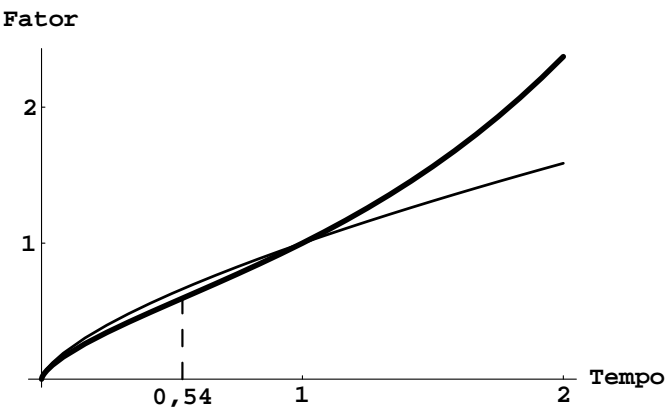

Figura 10. Fator de escala nos modelos de EdS (linha fina) e FL euclidiano (linha grossa), este com densidades atuais de $30 \%$ de matéria e $70 \%$ de energia escura.

Na Fig. 10 mostramos um gráfico do FL euclidiano, junto com o EdS para comparação. O ponto 0,54 no eixo horizontal indica a mudança da desaceleração inicial para a fase de aceleração em FL, quando o fator de escala tinha $65 \%$ do valor atual. Nossa Fig. 10 é a única baseada em cálculos exatos. Para os interessados nestes, o Apêndice traz as grandezas básicas e os valores adotados na Fig. 10.

Podemos agora dar uma idéia de como a aceleração atual foi descoberta. Para um dado redshift de uma supernova, a distância desta calculada no FL é maior do que no EdS. Portanto, seu brilho previsto é menor no primeiro caso - acelerado - e é o que corresponde ao brilho observado da supernova.

$\mathrm{Na}$ época 'atual' (em cosmologia isto pode significar um período de milhares ou até milhões de anos), temos a estimativa de uma densidade relativa de matéria de $30 \%$, e de energia escura dos restantes $70 \%$ - de modo a satisfazer a idéia dominante entre os teóricos, de que o período inflacionário (v. seção III acima) implica $\Omega_{\text {total }}$ $=1$, isto é, a densidade total de matéria e energia seria a densidade crítica. Por que na época atual esses valores são da mesma ordem de grandeza? Este é um dos chamados problemas de coincidência. O chamado princípio antrópico explica essa coincidência dizendo que só nessas condições do universo a vida humana poderia ter aparecido e se desenvolvido. Portanto, já que o ser humano existe, as densidades de matéria e do 
vácuo têm de ser da mesma ordem de grandeza. Eu não estou convencido de que esta é uma boa saída...

Uma outra idéia, alternativa à constante cosmológica, para explicar a aceleração, é postular um novo tipo de matéria, chamado quintessência, numa referência poética aos quatro elementos da antiguidade. Sua densidade poderia ser não constante, mas diminuir vagarosamente, de modo a simular uma constante na época atual, e se anular no futuro remoto.

\section{Nomenclatura e outros mode- los}

Em todos os casos acima, o espaço foi suposto isotrópico: em cada ponto suas propriedades são as mesmas em qualquer direção; e homogêneo: tem as mesmas propriedades em cada ponto. A classe dessas soluções chama-se modelos de Robertson-Walker. Destes, aqueles com a propriedade BB formam a subclasse FLRW (Friedmann-Lemaitre-RobertsonWalker). Mas essa nomenclatura não é uniforme na literatura especializada.

Não discuti nossa pesquisa aqui no IFT, que envolve modelos de curvatura constante, mas com espaços finitos e de formas mais complexas que os acima. Também não mencionei outros casos que são de grande interesse teórico, mas que no momento têm pouca relevância na cosmologia observacional.

\section{Conclusão}

O Big Bang, agora na versão de Friedmann-Lemaitre com constante cosmológica positiva e geometria espacial euclidiana, acomoda bem seus antigos sucessos. Estes incluem a expansão do universo, a formação e abundância, com relação ao próton, dos núcleos atômicos leves - dêuteron, hélio 3 , hélio 4 , lítio 7 - e a radiação cósmica de fundo. $\mathrm{E}$ também explica a atual possibilidade da aceleração da expansão.

Mas há um sentimento de incompleteza da teoria, que precisa apelar, por exemplo, para o princípio antrópico e para a misteriosa constante cosmológica, que ninguém sabe de onde vem. Há também o problema de conciliar a cosmologia com outras áreas da física, como a teoria quântica dos campos. Aparentemente, as teorias do cosmo encontram-se em uma fase transitória, à espera da elaboração de uma nova síntese teórica.

\section{Apêndice}

Modelo FL euclidiano, com os valores adotados na Fig. 10:

Constante de Hubble
Constante cosmológica
Densidade de matéria
Densidade de energia escura
Parâmetro de desaceleração
Métrica

Idade do universo

Distância própria

Transição desaceleração $\rightarrow$ aceleração

No modelo EdS, $a(t)=\left(t / t_{0}\right)^{2 / 3}, t_{0}=2 / 3 H_{0}=$ $10,0 \times 10^{9}$ anos, e $d(Z)=\left(2 c / H_{0}\right)\left[1-(1+Z)^{-1 / 2}\right]$. Esta é menor que sua correspondente no FL, para qualquer redshift; por exemplo, para $Z=1,0$, a razão dessas distâncias é 0,76 .

$$
\begin{aligned}
& H_{0}=65 \mathrm{~km} \mathrm{~s}^{-1} \mathrm{Mpc}^{-1} \\
& \Lambda=1,0368 \times 10^{-56} \mathrm{~cm}^{-2} \\
& \Omega_{M 0}=8 \pi G \rho_{M 0} / 3 H_{0}^{2}=0,3 \\
& \Omega_{\Lambda}=\Lambda c^{2} / 3 H_{0}^{2}=1-\Omega_{M 0}=0,7 \\
& q_{0}=\Omega_{M 0} / 2-\Omega_{\Lambda}=-0,55 \\
& d s^{2}=c^{2} d t-a^{2}(t) d \mathbf{x}^{2} \text {, onde } \\
& a(t)=\left(\Omega_{M 0} / \Omega_{\Lambda}\right)^{1 / 3} \sinh ^{2 / 3}(\sqrt{3 \Lambda} c t / 2) \\
& =0,754 \sinh ^{2 / 3}\left(1,210 t / t_{0}\right) \\
& t_{0}=\left(2 / 3 H_{0} \sqrt{\Omega_{\Lambda}}\right) \tanh ^{-1} \sqrt{\Omega_{\Lambda}} \\
& =14,5 \times 10^{9} \text { anos } \\
& d(Z)= \\
& \left(c / H_{0}\right) \int_{0}^{Z}\left[\Omega_{M 0}(1+z)^{3}+\Omega_{\Lambda}\right]^{-1 / 2} d z \\
& t_{d a}=(2 / \sqrt{3 \Lambda}) \tanh ^{-1}(1 / \sqrt{3}) \\
& =0,544 t_{0} \\
& Z_{d a}=\left(2 \Omega_{\Lambda} / \Omega_{M 0}\right)^{1 / 3}-1=0,671
\end{aligned}
$$

\section{Bibliografia - em ordem de dificul- dade crescente}

\author{
S. Jodra, La Tyrannie de l'Energie Sombre, em Ciel et \\ Espace, março/2001 \\ E. R. Harrison, Cosmology: the Science of the Universe
}


(Cambridge University Press, 1981)

R. D'Inverno, Introducing Einstein's Relativity (Oxford University Press, 1995)

H. C. Ohanian, Gravitation and Spacetime (W. W. Norton \& Co., Nova Iorque, 1976)

S. M. Carroll, Dark Energy and the Preposterous Universe, preprint arXiv/astro- ph/0107571
A. V. Filippenko, Einstein's Biggest Blunder? HighRedshift Supernovae and the Accelerating Universe, preprint arXiv/astro-ph/0109399

A. G. Riess e outros, The Farthest Known Supernova: Support for an Accelerating Universe and a Glimpse at the Epoch of Deceleration, preprint arXiv/astro$\mathrm{ph} / 0104455$ 